\title{
Line-profile variations of stochastically excited oscillations in four evolved stars *
}

\author{
S. Hekker ${ }^{1,2,3}$ and C. Aerts ${ }^{2,4}$ \\ 1 School of Physics and Astronomy, University of Birmingham, Edgbaston, Birmingham B15 2TT, UK \\ e-mail: saskia@bison.ph.bham.ac.uk \\ 2 Instituut voor Sterrenkunde, Katholieke Universiteit Leuven, Celestijnenlaan 200 D, 3001 Leuven, Belgium \\ 3 Royal Observatory of Belgium, Ringlaan 3, 1180 Brussels, Belgium \\ 4 Department of Astrophysics, IMAP, University of Nijmegen, PO Box 9010, 6500 GL Nijmegen, The Netherlands \\ Received 29 June 2009 / Accepted 8 February 2010
}

\section{ABSTRACT}

\begin{abstract}
Context. Since solar-like oscillations were first detected in red-giant stars, the presence of non-radial oscillation modes has been debated. Spectroscopic line-profile analysis was used in the first attempt to perform mode identification, which revealed that non-radial modes are observable. Despite the fact that the presence of non-radial modes could be confirmed, the degree or azimuthal order could not be uniquely identified. Here we present an improvement to this first spectroscopic line-profile analysis.

Aims. We aim to study line-profile variations in stochastically excited solar-like oscillations of four evolved stars to derive the azimuthal order of the observed mode and the surface rotational frequency.

Methods. Spectroscopic line-profile analysis is applied to cross-correlation functions, using the Fourier parameter fit method on the amplitude and phase distributions across the profiles.

Results. For four evolved stars, $\beta$ Hydri (G2IV), $\epsilon$ Ophiuchi (G9.5III), $\eta$ Serpentis (K0III) and $\delta$ Eridani (K0IV) the line-profile variations reveal the azimuthal order of the oscillations with an accuracy of \pm 1 . Furthermore, our analysis reveals the projected rotational velocity and the inclination angle. From these parameters we obtain the surface rotational frequency.

Conclusions. We conclude that line-profile variations in cross-correlation functions behave differently for different frequencies and provide additional information about the surface rotational frequency and azimuthal order.
\end{abstract}

Key words. stars: oscillations - stars: individual: $\epsilon$ Ophiuchi - stars: individual: $\eta$ Serpentis - stars: individual: $\delta$ Eridani - stars: individual: $\beta$ Hydri - line: profiles

\section{Introduction}

After several previous claims, the first firm observational evidence of solar-like oscillations in red-giant stars was presented by Frandsen et al. (2002) for $\xi$ Hydrae. This was followed by discoveries from two-site ground-based spectroscopic campaigns targeting $\epsilon$ Ophiuchi (De Ridder et al. 2006) and $\eta$ Serpentis (Barban et al. 2004). Based on theory of more luminous red giants (Dziembowski et al. 2001), the detected frequencies of these stars were interpreted as radial modes and the stars were modelled based on this assumption (Houdek \& Gough 2002; De Ridder et al. 2006).

Hekker et al. (2006) performed the first time-series analysis of spectral line shape variations and attempted to perform spectroscopic mode identification of the observed frequencies for three red-giant stars ( $\epsilon$ Ophiuchi, $\eta$ Serpentis and $\xi$ Hydrae) and one subgiant ( $\delta$ Eridani). They investigated line-profile variations in the cross-correlation functions based on a pixel-by-pixel method, i.e., fitting a sinusoid at every velocity pixel across the profile. From the differences in the shape of the amplitude distribution across the profile for different frequencies they concluded that non-radial oscillations must be present, although no definite identification could be provided.

The CoRoT (Convection Rotation and planetary Transits) satellite performs photometry on parts of the sky for 150

^ The software package FAMIAS developed in the framework of the FP6 European Coordination Action HELAS

(http://www.helas-eu.org) has been used in this research. consecutive days with $32 / 512$ s cadences in the centre and anti-centre direction of the galaxy. Among the observed stars are many red giants. With these observations the existence of non-radial oscillations in red-giant stars has been firmly proven (De Ridder et al. 2009). This proof is based on the determination of the harmonic degrees, using the asymptotic relation for high-order low-degree modes (Tassoul 1980). This relation predicts that non-radial modes appear at regular intervals in the Fourier spectrum (large separation) with modes of harmonic degree $\ell=1$ approximately half way in between the radial modes and modes with harmonic degree $\ell=2$ close to the radial modes (small separation). Indeed, multiple "ridges" are present in échelle diagrams (frequency versus frequency modulo large separation) of several stars presented by De Ridder et al. (2009). These results have been confirmed by observations of red giants from the NASA Kepler satellite, see e.g., Bedding et al. (2010), and will be followed up.

The asymptotic approximation is valid for $\mathrm{p}$ modes and can only be applied to giants and subgiants when the oscillation modes are trapped in the outer parts of the star. The trapping depends on the internal stellar structure. For stars in certain evolutionary states, it is inefficient, such that g modes and mixed modes are also observable, see e.g., Dupret et al. (2009). This degrades the regular pattern, and the asymptotic relation is less useful as a guide for performing identification of the mode degree $\ell$.

For non-radial solar-like oscillations it is expected that all azimuthal orders are excited. However, it is usually not possible to 
distinguish between oscillation frequencies of modes with the same radial order $(n)$, harmonic degree $(\ell)$, and different azimuthal order $(m)$, due to the generally slow rotational velocity of evolved solar-like oscillators, the limited time span of observations, and the stochastic nature of the oscillations. Slow rotation induces only a very small frequency splitting, while the stochastic nature causes the oscillation frequencies to appear as Lorentzian profiles in the Fourier spectrum with a width that depends on the lifetime of the mode, i.e., shorter mode lifetimes correspond to broader profiles. We need long time series of data to obtain sub- $\mu \mathrm{Hz}$ frequency resolution and to resolve the modes, which can have lifetimes of the order of a few to hundreds of days (Dupret et al. 2009). So, the frequency of a non-radial oscillation mode is extracted from a broad profile consisting of several modes with different $m$ values. Which of these modes is dominant depends on the inclination angle. In stars seen at high inclination angle, the $m \neq 0$ modes have higher visibility than $m=0$ modes (Gizon \& Solanki 2003). Furthermore, for individual realisations when we consider a single time series of observations, the stochastic interference with noise might boost or diminish a mode. Therefore, we are in principle able to observe any of the present azimuthal orders or combinations thereof. The combination of different orders will also behave differently from $m=0$ modes due to the influence of $m \neq 0$ modes.

Line-profile variations are sensitive mainly to the azimuthal order of a mode and much less sensitive to its harmonic degree (see e.g., Aerts \& Eyer 2000). The determination of a non-zero azimuthal order would indicate that the oscillation mode is nonradial. This can be important in cases where the asymptotic approximation fails or provides ambiguous results, i.e., where different ridges in an échelle diagram cannot be unambiguously identified. When $\ell$ is known from the asymptotic approximation the $m$ value can place additional constraints on the internal structure. Also, we can obtain the inclination angle for non-radial modes and the projected rotational velocity from line-profile analyses. These parameters allow us to determine the stellar rotational frequency for non-radial oscillators.

With this potential of the analysis of line-profile variations in mind, we have improved the spectroscopic line-profile analysis originally presented by Hekker et al. (2006) (Sect. 3). These improvements were necessary as Hekker et al. (2006) were only able to match observations and theory qualitatively. In this work, we perform a quantitative analysis. We use FAMIAS (Frequency Analysis and Mode Identification for Asteroseismology, Zima 2008), a package of state-of-the-art tools for the analysis of photometric and spectroscopic time-series data, to perform lineprofile analyses (Sect. 4) on three stars, $\beta$ Hydri (G2IV) $\delta$ Eridani (K0IV) and $\epsilon$ Ophiuchi (G9.5III), for which previous mode identification was available (Bedding et al. 2007; Carrier 2002; Carrier et al. 2003; Kallinger et al. 2008).

In Sect. 5, we analyse the observed line-profile variations for oscillation modes of $\beta$ Hydri, $\delta$ Eridani, and $\epsilon$ Ophuichi. For non-radial modes in these stars, as identified from the asymptotic approximation, we obtain the azimuthal orders and a constraint on the inclination angle. The latter combined with the projected rotational velocity gives an estimate of the stellar rotational frequency.

The results of the line-profile analyses of $\beta$ Hydri, $\delta$ Eridani, and $\epsilon$ Ophiuchi gave us confidence that we can perform a quantitative comparison between line-profile variations from observations and synthetic spectra, which provides the azimuthal order and for non-radial oscillators, the inclination angle and with that the surface rotational frequency. Therefore, we perform the same analysis for $\eta$ Serpentis (K0III), for which no previous mode identification was available (Sect. 5).

\section{Observations}

For $\epsilon$ Ophiuchi and $\eta$ Serpentis, we have spectra from the fiberfed échelle spectrograph CORALIE mounted on the Swiss $1.2 \mathrm{~m}$ Euler telescope at La Silla (ESO, Chile) at our disposal. These were obtained during a two-site campaign using CORALIE and ELODIE, the fiber-fed spectrograph mounted on the French $1.93 \mathrm{~m}$ telescope at Observatoire de Haute Provence, France, during the summer of 2003. The ELODIE spectra are available, but their cross-correlation profiles have a resolution of $1 \mathrm{~km} \mathrm{~s}^{-1}$, which is coarse compared to the $0.1 \mathrm{~km} \mathrm{~s}^{-1}$ resolution of the CORALIE spectra, and hence are not used for the present analysis. The observations of $\delta$ Eridani were taken with CORALIE during a twelve-day campaign in November 2001. For $\beta$ Hydri, we use spectra obtained with the fiber-fed échelle spectrograph HARPS mounted on the $3.6 \mathrm{~m}$ telescope at La Silla (ESO, Chile), which were obtained during a two-site campaign using HARPS and UCLES, the échelle spectrograph mounted on the $3.9 \mathrm{~m}$ Anglo-Australian Telescope, Siding Spring Observatory, Australia, in September 2005. Spectra from UCLES are contaminated with iodine absorption lines, used to obtain accurate radial velocity variations, and are therefore not useful for the present analysis. For a detailed description of the data, we refer to the publications describing the observations of solarlike oscillations in these stars, De Ridder et al. (2006), Barban et al. (2004), Carrier (2002), Carrier et al. (2003), Bedding et al. (2007), respectively. Stellar parameters of the four stars are listed in Table 1.

We note here that we have data for $\xi$ Hydrae at our disposal, but as already shown by Hekker et al. (2006) the signal-to-noise ratio $(\mathrm{S} / \mathrm{N})$ of the oscillations is too low for the present analysis and therefore we did not include $\xi$ Hydrae in the current investigation.

\section{Improved analysis}

Hekker et al. (2006) used the INTER-TACOS (INTERpreter for the Treatment, the Analysis and the COrrelation of Spectra) software package developed at Geneva Observatory (Baranne et al. 1996) to compute cross-correlation functions (CCFs) of the observed spectra. The CCFs are computed using a mask containing absorption lines of a K0 star for $\epsilon$ Ophiuchi, $\eta$ Serpentis, and $\delta$ Eridani and have a resolution of $0.1 \mathrm{~km} \mathrm{~s}^{-1}$. For the HARPS data of $\beta$ Hydri, we use the CCFs computed with the HARPS pipeline (Rupprecht et al. 2004), which uses a mask containing absorption lines of a G2 star. These CCFs have a resolution of $0.5 \mathrm{~km} \mathrm{~s}^{-1}$.

Here we use these CCFs as a starting point of our lineprofile analysis but we apply three corrections before attempting to analyse the line-profile variations. These corrections are necessary, as effects that are not intrinsic to the star (instrumental effects/changing weather conditions) cause additional changes in the line profiles on a night by night basis. These effects hampered a quantitative analysis by Hekker et al. (2006). The following corrections are applied to isolate the line-profile variations caused by oscillations from extrinsic variations:

- The first correction concerns a continuum normalisation. Continuum on both sides of each CCF is selected and a linear polynomial is fitted through these continuum points. The full cross-correlation profile is then divided by this linear fit. 
S. Hekker and C. Aerts: Line-profile variations of stochastically excited oscillations in evolved stars

Table 1. Stellar parameters of $\epsilon$ Ophiuchi, $\eta$ Serpentis, $\delta$ Eridani, and $\beta$ Hydri.

\begin{tabular}{lcccc}
\hline \hline parameter & $\epsilon$ Ophiuchi & $\eta$ Serpentis & $\delta$ Eridani & $\beta$ Hydri \\
\hline$T_{\text {eff }}[\mathrm{K}]$ & $4970 \pm 80^{a}$ & $4955 \pm 80^{a}$ & $5050 \pm 100^{h}$ & $5872 \pm 44^{j}$ \\
$R\left[R_{\odot}\right]$ & $10.4 \pm 0.45^{b+d, c}$ & $5.914 \pm 0.094^{b+d}$ or $5.794 \pm 0.097^{f}$ & $2.33 \pm 0.03^{i}$ & $1.814 \pm 0.017^{j}$ \\
$M\left[M_{\odot}\right]$ & $2.0^{c}$ & $2.42 \pm 0.2^{f}$ or $1.72_{-0.11}^{+0.15 g}$ & $1.1215^{i}$ & $1.07 \pm 0.03^{j}$ \\
{$[\mathrm{Fe} / \mathrm{H}]$} & $-0.07 \pm 0.11^{a}$ & $-0.15 \pm 0.11^{a}$ & $0.13 \pm 0.03^{h}$ & $-0.2^{k}$ \\
$\log g(\mathrm{c} . \mathrm{g} . \mathrm{s})$. & $2.9 \pm 0.15^{a}$ & $3.2 \pm 0.15^{a}$ & $3.77 \pm 0.16^{h}$ & $3.952 \pm 0.005^{j}$ \\
$v \sin i\left[\mathrm{~km} \mathrm{~s}^{-1}\right]$ & $3.5 \pm 0.5^{a}$ & $0.44 \pm 0.44^{a}$ & $2.3 \pm 0.5^{e}$ & $3.3 \pm 0.3^{e}$ \\
$\xi_{\text {micro }}\left[\mathrm{km} \mathrm{s}^{-1}\right]$ & $1.5 \pm 0.1^{a}$ & $1.3 \pm 0.1^{a}$ & & \\
$\xi_{\text {macro }}\left[\mathrm{km} \mathrm{s}^{-1}\right]$ & $3.55 \pm 0.45^{a}$ & $3.52 \pm 0.45^{a}$ & & \\
$\pi[\mathrm{mas}]$ & $30.34 \pm 0.79^{d}$ & $52.81 \pm 0.75^{d}$ & $110.58 \pm 0.88^{d}$ & $133.78 \pm 0.5^{d}$ \\
$m_{v}[\mathrm{mag}]$ & $3.24 \pm 0.02^{d}$ & $3.23 \pm 0.02^{d}$ & $3.52 \pm 0.02^{d}$ & $2.82 \pm 0.02^{d}$ \\
\hline
\end{tabular}

Notes. Effective temperature $\left(T_{\text {eff }}\right)$ in Kelvin, radius $(R)$ in $R_{\odot}$, mass $(M)$ in $M_{\odot}$, metallicity $([\mathrm{Fe} / \mathrm{H}])$, surface gravity $(\log g)$ in c.g.s units, rotational velocity $(v \sin i)$, microturbulence $\left(\xi_{\text {micro }}\right)$ and macroturbulence $\left(\xi_{\text {macro }}\right)$ in $\mathrm{km} \mathrm{s}^{-1}$, parallax $(\pi)$ in mas, and apparent magnitude in the $V$ band $\left(m_{v}\right)$. References. ${ }^{(a)}$ Hekker \& Meléndez (2007); ${ }^{(b)}$ Richichi et al. (2005); ${ }^{(c)}$ Kallinger et al. (2008); ${ }^{(d)}$ Perryman \& ESA (1997); ${ }^{(e)}$ Reiners \& Schmitt (2003); ${ }^{(f)}$ Valenti \& Fischer (2005); ${ }^{(g)}$ Valenti \& Fischer (2005) isochrone stellar mass; ${ }^{(h)}$ Santos et al. (2004); ${ }^{(i)}$ Thévenin et al. (2005); ${ }^{(j)}$ North et al. (2007); ${ }^{(k)}$ Dravins et al. (1998).
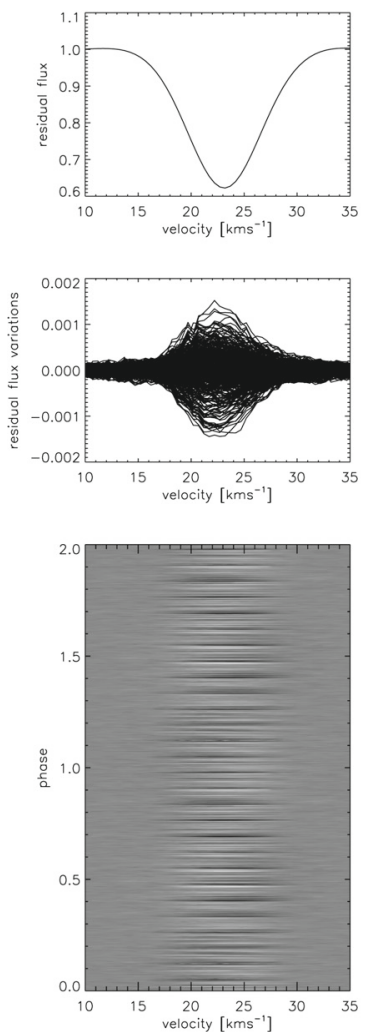
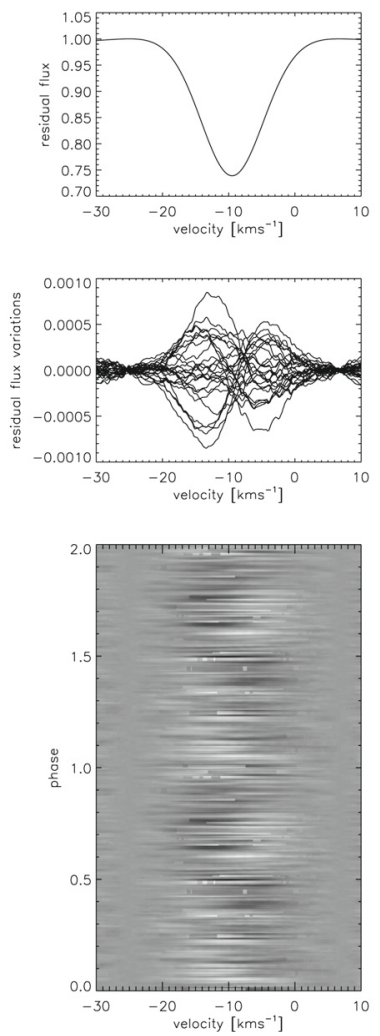
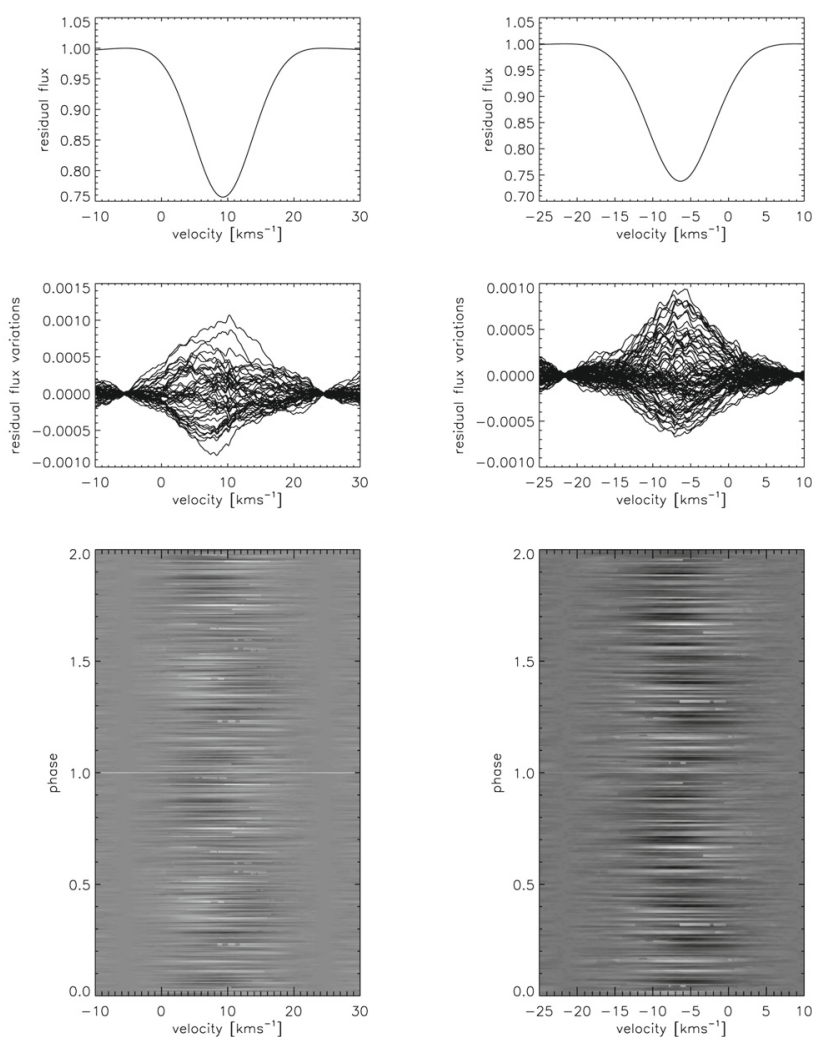

Fig. 1. Average cross-correlation functions (top), isolated line-profile variations after subtraction of the average CCF (middle, only one night is displayed for clarity), and grayscale plots of the isolated line-profile variations as a function of phase for the respective dominant frequencies, where darker regions indicate negative values, while lighter regions indicate positive values (bottom). From left to right, $\beta$ Hydri, $\epsilon$ Ophiuchi, $\eta$ Serpentis, and $\delta$ Eridani.

- The second correction involves the isolation of the lineprofile variations and removal of outliers. For each observing night we calculate for each velocity bin the average $\mathrm{CCF}$ value and its standard deviation. In each bin the average $\mathrm{CCF}$ is subtracted from the observed continuum-corrected $\mathrm{CCF}$ to isolate the variations. Outliers are identified as values that deviate from the average CCF by more than three times the standard deviation. CCFs with outliers in one or more velocity bins are fully discarded.

- Finally, the flux variations in each velocity bin are imposed on the mean CCF of all observations. This is not strictly necessary to perform the line-profile analysis, but we aim to use moments to obtain the oscillation frequencies and therefore we need to have a line profile rather than the residual profile.

The resulting average CCFs and isolated line-profile variations for all four stars are shown in Fig. 1 together with grayscale plots of the isolated line-profile variations.

Extensive tests have been performed to investigate the influence of these corrections and removal of outliers, in particular to confirm that these indeed remove effects that are not intrinsic to 

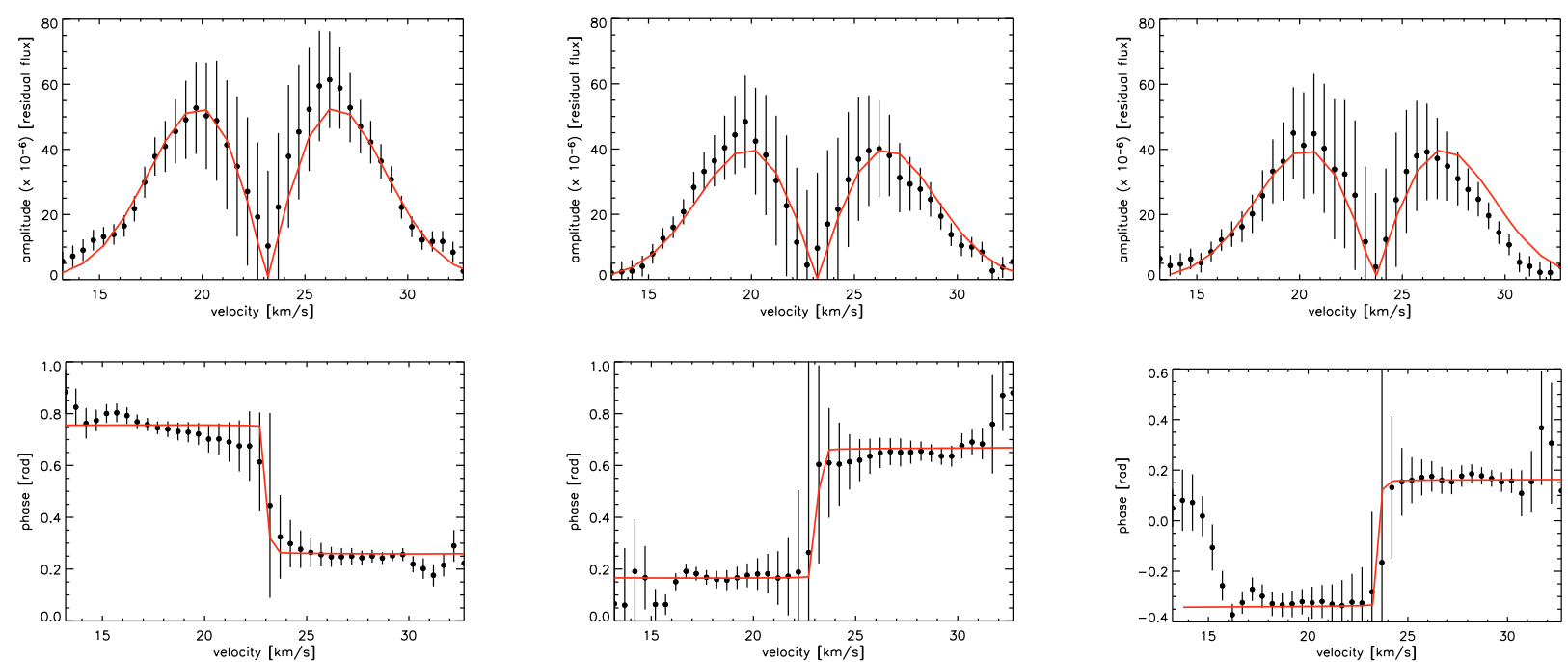

Fig. 2. Amplitude (top) and phase (bottom) distributions as a function of velocity across the line profile of $\beta$ Hydri for the three radial modes with frequencies obtained from the first moment $\langle\mathrm{v}\rangle, v_{\langle\mathrm{v}\rangle}=86.8 \mathrm{c} \mathrm{d}^{-1}(1004.4 \mu \mathrm{Hz})($ left $), v_{\langle\mathrm{v}\rangle}=73.0 \mathrm{c} \mathrm{d}^{-1}(845.0 \mu \mathrm{Hz})($ centre $)$, and $v_{\langle\mathrm{v}\rangle}=96.7 \mathrm{c} \mathrm{d}-1$ $(1118.9 \mu \mathrm{Hz})($ right $)$. The mean radial velocity of the star is approximately $23.2 \mathrm{~km} \mathrm{~s}^{-1}$. The data are indicated with black dots and the $(\ell, m)=(0,0)$ fit is indicated in red solid line (colours are given only in the online version). In cases where the observed distributions are not centred around the midpoint of the CCF, the fits are shifted (see Sect. 4, for further explanation).

the star and do not influence the intrinsic behaviour. These tests indicate that the results presented here are robust to degrading the signal and to changes in the average profiles during the night due to for instance varying weather conditions or instrumental effects.

With FAMIAS (Zima 2008), we compute frequencies from the first moment (Aerts et al. 1992) for the new CCFs, similar to what was performed by Hekker et al. (2006) for the uncorrected CCFs. In general, we recover the same frequencies (or 1 day aliases) as Hekker et al. (2006) for $\epsilon$ Ophiuchi, $\eta$ Serpentis and as Bedding et al. (2007), Carrier (2002), Carrier et al. (2003) for $\beta$ Hydri and $\delta$ Eridani, respectively.

\section{Line-profile analysis}

The line-profile analysis is performed using the Fourier Parameter Fit (FPF) method (Zima 2006). The FPF method relies on a fit to the observational Fourier parameters across the line profile. For every detected pulsation frequency, the zero point, amplitude, and phase are computed for every velocity bin across the profile and compared with the same quantities from synthetic profiles. This procedure relies only on the velocity eigenfunction at the stellar surface and is therefore model independent (see Chapter 6 of Aerts et al. 2009). We use the standard sign convention for the azimuthal order $m$ in FAMIAS, i.e., $m>0$ implies prograde modes.

One has to keep in mind that this method is mainly sensitive to the azimuthal order $(m)$ of the mode and far less to its harmonic degree $(\ell)$, as modes with the same order but similar degree will give essentially the same amplitude and phase distributions (Zima 2006).

For the present analysis, we use the frequencies of the first moment of the new CCFs and the stellar parameters listed in Table 1 as input parameters. We first computed pulsationally independent parameters, i.e., equivalent width $(E W)$, macroturbulent broadening $\left(\xi_{\text {macro }}\right)$, projected rotational velocity $(v \sin i)$, and radial velocity (RV) and then fix these parameters. The inclination angle is a free parameter in all calculations. Then we use the FPF method to fit the amplitude and phase distributions either for modes for which we know the degree from previous determinations or for a grid of modes with degree $0 \leq \ell \leq 4$, and order $-\ell \leq m \leq \ell$. Modes of up to degree 4 are investigated as in spectroscopy the partial cancellation effect would theoretically allow this. For examples of amplitude and phase distributions, see for example Fig. 2.

Although we investigate stochastic oscillations, we do not include damping and excitation in the synthetic profiles but take the stochastic effects into account in the interpretation of the results. The main reason for this is that we know that damping and excitation can cause asymmetries in the amplitude and phase distribution (Hekker et al. 2006), but the exact nature of these asymmetries varies between realisations. In their Fig. 10, Hekker et al. (2006) show examples of amplitude and phase distributions of simulated noise-free line profiles with a two-day damping time. For each of the modes $\ell=0,1,2$ and $m=0-\ell$, they show 10 realisations. From these simulations, it is clear that for different realisations of the stochastically damped and re-excited oscillations, the height of the amplitude distributions varies for all modes. In addition, the centroids of the distributions for modes with $m \neq 0$ can be shifted away from the laboratory wavelength. The asymmetries in the amplitude profiles are most pronounced for sectorial modes with $\ell=|m|$, but are also present for other $m \neq 0$ modes. Moreover, from simulations of spectra with signal-to-noise ratios of 50, 100, and 150, we conclude that the asymmetries in the amplitude distribution are also present for modes with $m=0$. When interpreting our results, we take the asymmetries and shifts into account by shifting the computed amplitude distributions such that their central minima overlap with the central minimum of the observed distribution. The same shift is applied to the computed phase distributions.

To identify the best fit to the data, $\chi^{2}$ values are computed, as foreseen in FAMIAS. These are used to select the fits with $\chi^{2}$ below 1 . However, it turned out that a reasonably large number of profiles satisfy this requirement and that among these best fit solutions the optimum $m$ value can differ when investigating only the central part of the line, or when the full line profile is taken into account. Therefore, we discuss the results of our line-profile modelling from visual inspection of the amplitude and phase 
S. Hekker and C. Aerts: Line-profile variations of stochastically excited oscillations in evolved stars
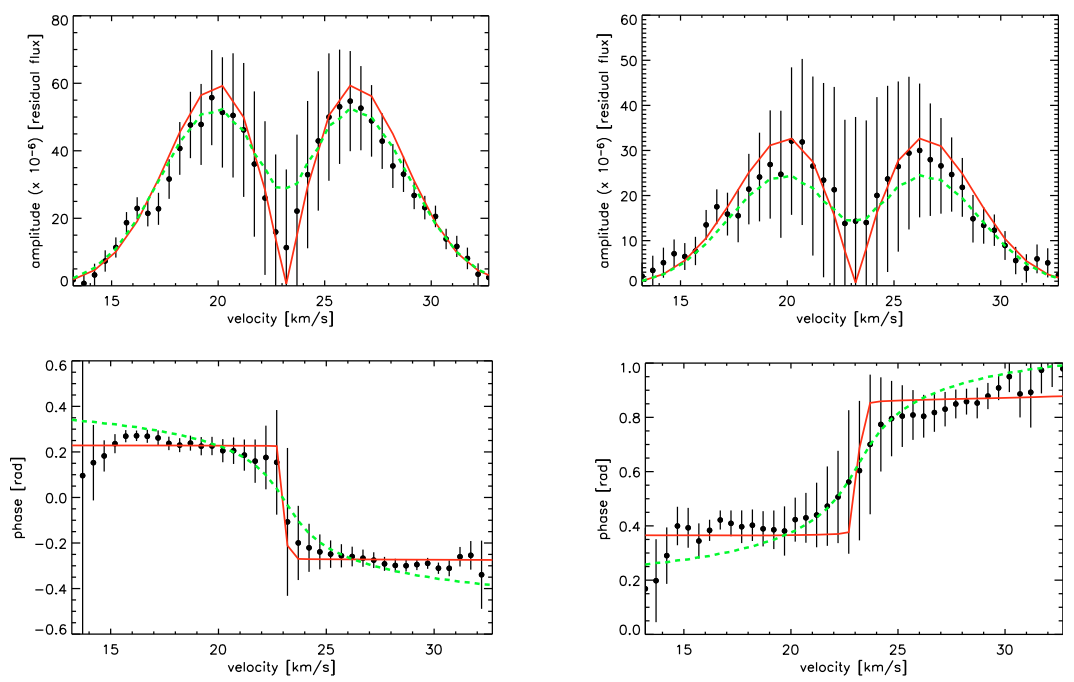

Fig. 3. Same as Fig. 2 for two frequencies obtained for $\beta$ Hydri from $\langle\mathrm{v}\rangle, v_{\langle\mathrm{v}\rangle}=89.3 \mathrm{c} \mathrm{d}^{-1}(1033.1 \mu \mathrm{Hz})(l e f t), v_{\langle\mathrm{v}\rangle}=103.0 \mathrm{c} \mathrm{d}-1(1192.3 \mu \mathrm{Hz})$ (right), for which the harmonic degrees have been determined previously to be $\ell=1$. In these panels, the $(\ell, m)=(1,0)$ and $(\ell, m)=(1, \pm 1)$ fits are indicated with red solid and green dashed lines, respectively.

distributions across the line profiles as has been done previously in the literature (e.g., Telting et al. 1997; and Briquet et al. 2005, 2009 for $\beta$ Cephei, $\theta$ Ophiuchi, and HD180642, respectively).

\section{Results}

The analysis of line-profile variations for solar-like oscillations in evolved stars as discussed above is applied first on stars for which mode identification has already been performed, i.e., $\beta$ Hydri, $\delta$ Eridani, and $\epsilon$ Ophiuchi.

\section{1. $\beta$ Hydri}

For $\beta$ Hydri, the degrees of the modes with highest power are identified using the asymptotic relation (Tassoul 1980) by Bedding et al. (2007). From the HARPS data at our disposal, we obtain five frequencies in common with Bedding et al. (2007), or one-day aliases, for which we were able to analyse the line profile variations. For three common frequencies, Bedding et al. (2007) determined $\ell=0$ and for the other two modes $\ell=1$ was found. We use these five frequencies for the line profile analysis. The results of the best fits to the observed line-profile variations obtained with the FPF method are shown in Figs. 2 and 3. We list the equivalent width, projected rotational velocity $(v \sin i)$, and macro turbulence $\xi_{\text {macro }}$ for all four stars in Table 2. The $v \sin i$ that we obtain here for the line profile of $\beta$ Hydri is slightly higher than the independent determination by Reiners \& Schmitt (2003).

Inspection of the amplitude and phase distributions reveals clearly that for the radial modes the fits to the observed lineprofile variations are consistent within their errors (see Fig. 2). For the two modes with $\ell=1$, we fitted $-\ell \leq m \leq \ell$ and plot the fits for the zonal mode and modes with $m=1$ and $m=-1$ for oscillations with frequencies $1033.1 \mu \mathrm{Hz}$ and $1192.3 \mu \mathrm{Hz}$, respectively (see left and right panels of Fig. 3). For $v=1033.1 \mu \mathrm{Hz}$, the fit with $m=0$ seems to be the best fit, while for $\nu=1192.3 \mu \mathrm{Hz}$ the fit with $m=-1$ seems favourable over the $m=0$ fit. Nevertheless, for both frequencies the zonal and sectoral modes are consistent with the observations within the errors.

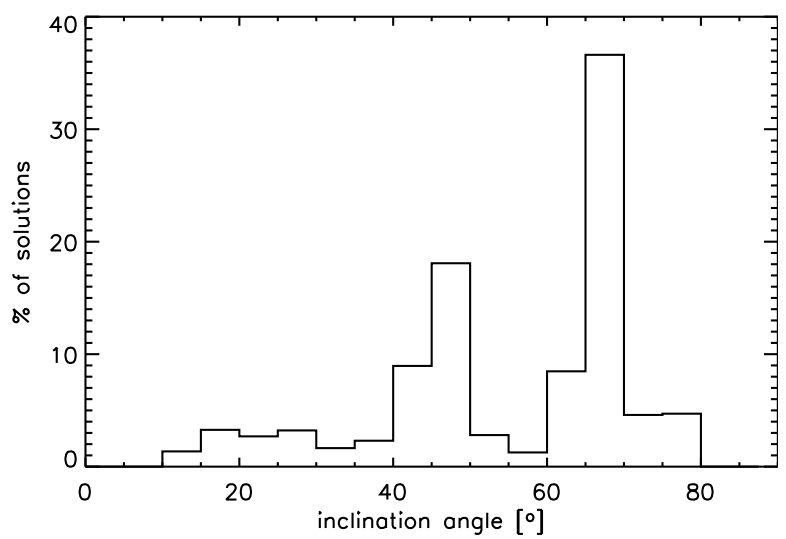

Fig. 4. Histogram of the inclination angles of all computed models for $\beta$ Hydri in which $\chi^{2}$ is used as a weight. See text for more details.

Table 2. Global line profile parameters as computed with FAMIAS and used for the line profile analysis.

\begin{tabular}{lcccc}
\hline \hline Parameter & $\epsilon$ Ophiuchi & $\eta$ Serpentis & $\delta$ Eridani & $\beta$ Hydri \\
\hline$E W\left[\mathrm{~km} \mathrm{~s}^{-1}\right]$ & 3.0 & 2.7 & 2.8 & 3.2 \\
$v \sin i\left[\mathrm{~km} \mathrm{~s}^{-1}\right]$ & 5.7 & 5.6 & 4.9 & 4.3 \\
$\xi_{\text {macro }}\left[\mathrm{km} \mathrm{s}^{-1}\right]$ & 3.7 & 3.4 & 3.6 & 2.6 \\
\hline
\end{tabular}

Notes. Terminology is similar to that used in Table 1.

Because of the non-radial nature of the latter two modes, the line profile analysis depends on the inclination angle of the star, which together with the projected rotational velocity infers a surface rotational frequency $(\Omega)$. For the inclination angle, we consider the interval defined by the weighted mean and standard deviation. The weight is defined as $w_{k}=\chi_{0}^{2} / \chi_{k}^{2}$, where $\chi_{0}^{2}$ is the $\chi^{2}$ of the best-fit solution (for more details see Desmet et al. 2009). For this star, we find an inclination of $55 \pm 17^{\circ}$. A histogram of the weighted inclination is shown in Fig. 4. So for $\beta$ Hydri, we find a surface rotational frequency ranging between $3.6 \mu \mathrm{Hz}$ and $5.5 \mu \mathrm{Hz}$ for inclination angles between $72^{\circ}$ and $38^{\circ}$ and a projected rotational velocity of $4.3 \mathrm{~km} \mathrm{~s}^{-1}$ as obtained with FAMIAS. 

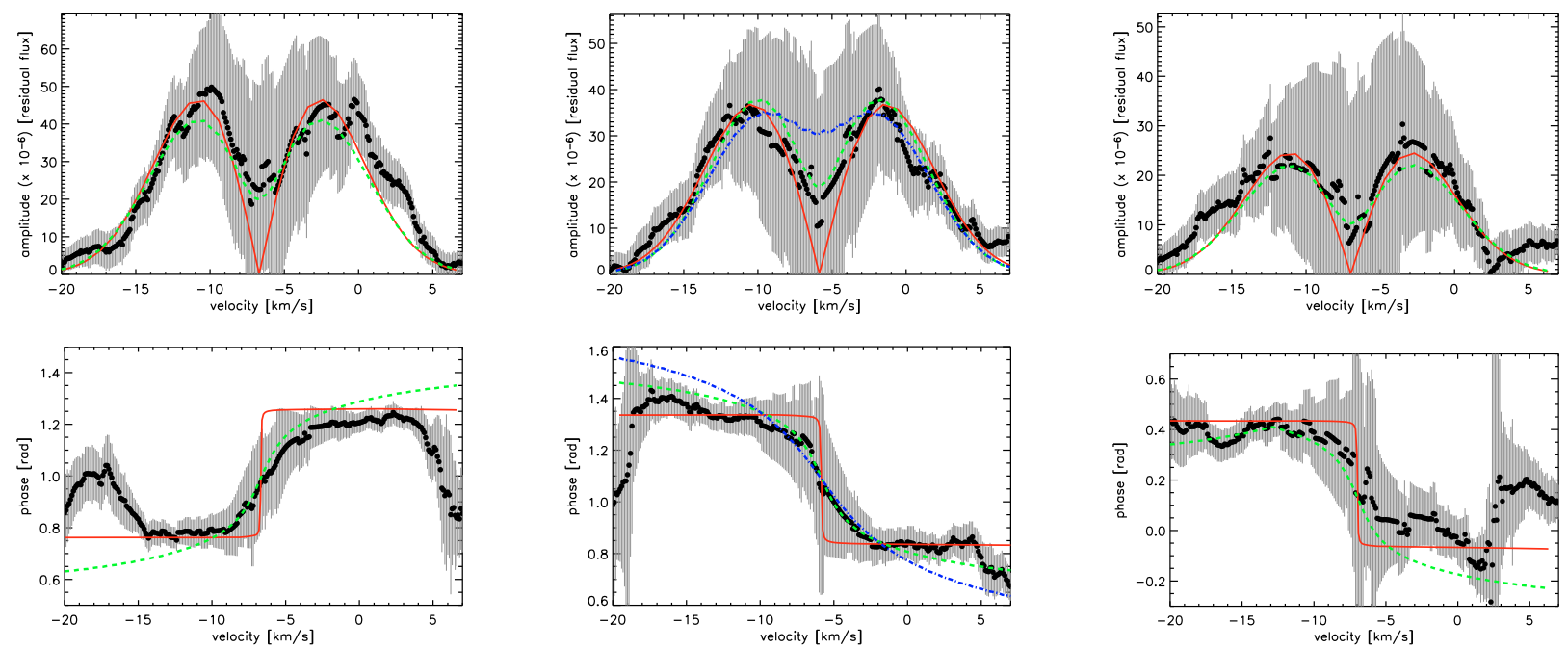

Fig. 5. Same as Fig. 2 for the significant frequencies of $\delta$ Eridani obtained from $\langle\mathrm{v}\rangle, v_{\langle\mathrm{v}\rangle}=58.4 \mathrm{c} \mathrm{d}^{-1}(675.8 \mu \mathrm{Hz})(l e f t), v_{\langle\mathrm{v}\rangle}=61.1 \mathrm{c} \mathrm{d}{ }^{-1}$ $(706.9 \mu \mathrm{Hz})($ centre $)$, and $\nu_{\langle\mathrm{v}\rangle}=57.5 \mathrm{c} \mathrm{d}^{-1}(665.9 \mu \mathrm{Hz})($ right $)$. The mean radial velocity of the star is approximately $-6.3 \mathrm{~km} \mathrm{~s}{ }^{-1}$. The fits with $m=0, \pm 1, \pm 2$ are indicated with red solid, green dashed and blue dotted lines, respectively.

\section{2. $\delta$ Eridani}

Carrier (2002), Carrier et al. (2003) analysed the radial velocity variations of $\delta$ Eridani using CORALIE observations and found evidence for 15 oscillation modes whose harmonic degrees could be identified using an échelle diagram. We obtain three frequencies in common (or one-day aliases) in the variations of the first moment of the same data set, with enough signal-to-noise ratio to analyse the line-profile variations. The harmonic degrees of all three oscillation frequencies are known and used as inputs for the present line-profile analysis. The results of this analysis are listed in Table 2 and shown in Fig. 5.

The most dominant mode we obtain from the first moment has a frequency of $675.8 \mu \mathrm{Hz}$ and was identified by Carrier (2002), Carrier et al. (2003) as a radial mode. Our fit of a radial mode, is indeed consistent with the data within errors. The fact that the "centre" part of the amplitude distribution does not decrease to 0 is an indication of the presence of a non-radial mode. A fit with $(\ell, m)=(1,-1)$ does provide a closer fit to the amplitude distribution and the centre part of the phase distribution (see left panels of Fig. 5).

The second frequency is identified by Carrier (2002), Carrier et al. (2003) as an $\ell=2$ mode, and from our present analysis we see that $(\ell, m)=(2,1)$ seems the most likely mode identification (see central panels of Fig. 5). The third frequency is consistent with $\ell=1$ as determined by Carrier (2002), Carrier et al. (2003), but we are unable to discriminate between $m=0$ or $m=-1$ due to the lower signal-to-noise ratio, which mostly influences the phase distribution of this frequency (see right panels of Fig. 5).

Also for this star, we investigated the weighted mean and standard deviation of all inclination angles of the computed synthetic profiles (see Fig. 6 for an histogram in which the weights are taken into account) to compute the surface rotational frequency. The inclination angle is $57 \pm 19^{\circ}$, which results in a range of surface rotational frequencies between 3.1 and $4.9 \mu \mathrm{Hz}$ at inclination angles ranging from 76 to $38^{\circ}$ and a projected rotational velocity of $4.9 \mathrm{~km} \mathrm{~s}^{-1}$.

\section{3. $\epsilon$ Ophiuchi}

De Ridder et al. (2006) and Barban et al. (2007) interpreted the frequencies observed in $\epsilon$ Ophiuchi, obtained from radial

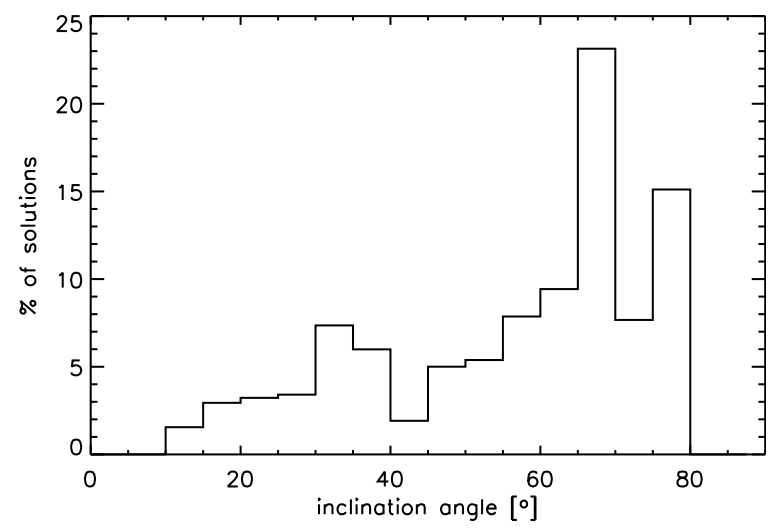

Fig. 6. Same as Fig. 4 for $\delta$ Eridani.

velocities derived from CORALIE and ELODIE data and from photometric space data (MOST), respectively, as radial modes. Based on the combined data set, Kallinger et al. (2008) interpreted the frequencies of $\epsilon$ Ophiuchi as both radial and nonradial oscillation modes. They performed mode identification by comparing the observed frequencies with those of theoretical models to derive the degree of the modes. Hence this mode identification is model dependent. From the (same) CORALIE data we have at our disposal, we obtain three frequencies of which only for one frequency, the harmonic degree of the oscillation was previously identified by Kallinger et al. (2008). The results of the best fits we obtain with the FPF method are listed in Table 2 and shown in Fig. 7.

A first inspection of the amplitude and phase distribution immediately shows that for all frequencies $m=-2$ provides the least likely mode identification.

For the 1st frequency, we find that fits for both $m=0$ and $m=-1$ are within the errors consistent with the amplitude distribution for the full CCF, where the $m=-1$ fit is superior in the centre of the line (see left top panel of Fig. 7). This is also the case in the centre of the phase distribution, where the $m=-1$ fit matches the observations closely, while the $m=0$ fit lies outside the error bars. In the wings of the CCF the opposite is true, i.e., the $m=0$ fit matches the observations and the $m=-1$ fit falls outside the error bars. Thus from the line centre, we would 
S. Hekker and C. Aerts: Line-profile variations of stochastically excited oscillations in evolved stars
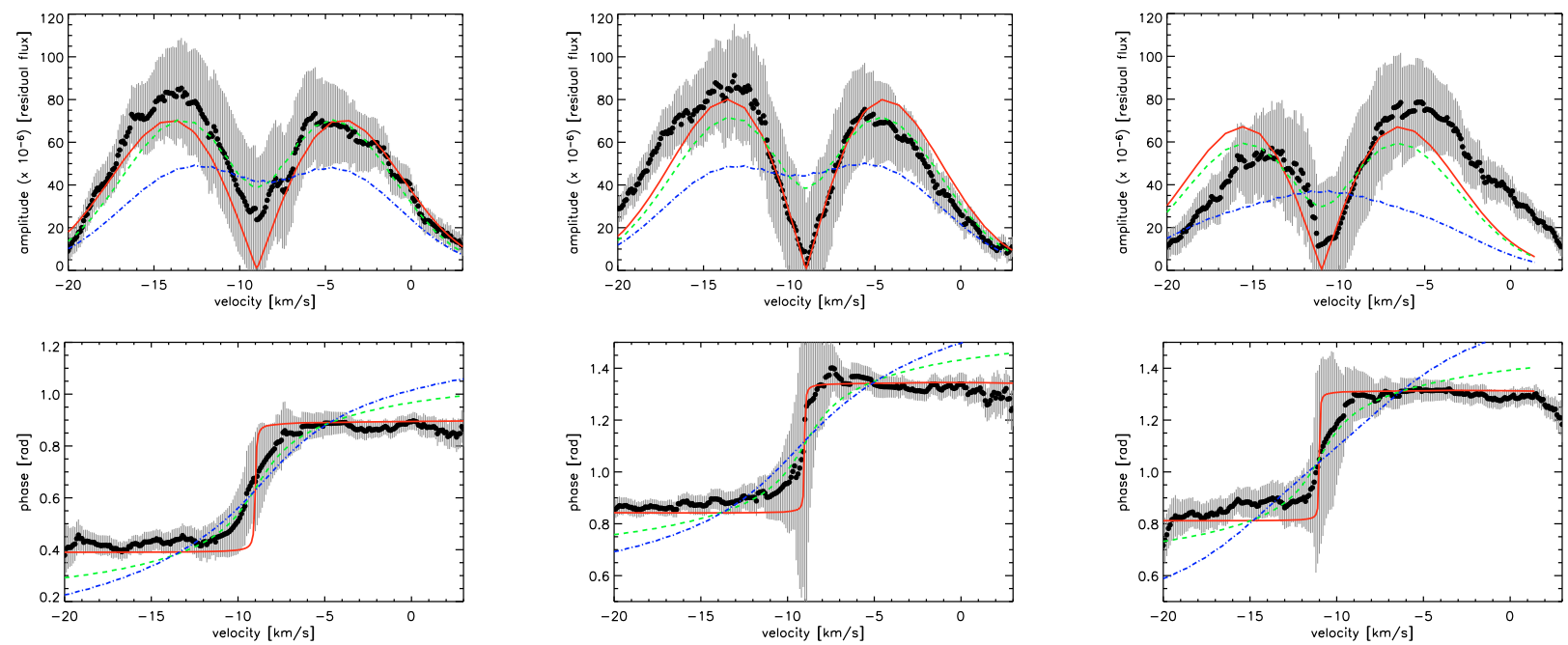

Fig. 7. Same as Fig. 5 for the significant frequencies of $\epsilon$ Ophiuchi obtained from $\langle\mathrm{v}\rangle: v_{\langle\mathrm{v}\rangle}=5.03 \mathrm{~cd}^{-1}(58.2 \mu \mathrm{Hz})(l e f t), v_{\langle\mathrm{v}\rangle}=4.65 \mathrm{~cd} \mathrm{~d}^{-1}$ $(53.8 \mu \mathrm{Hz})($ centre $)$ and $v_{\langle\mathrm{v}\rangle}=6.46 \mathrm{c} \mathrm{d}^{-1}(74.8 \mu \mathrm{Hz})($ right $)$. The mean radial velocity of the star is approximately $-9.4 \mathrm{~km} \mathrm{~s}^{-1}$.

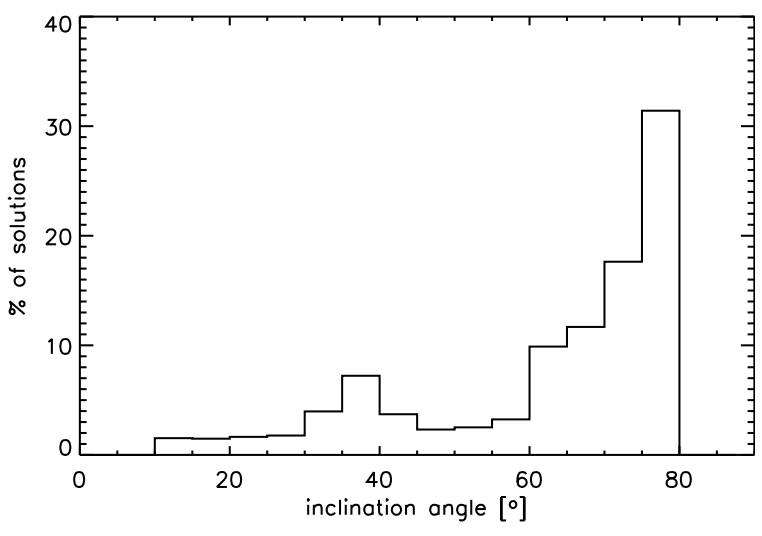

Fig. 8. Same as Fig. 4 for $\epsilon$ Ophiuchi.

conclude that $m=-1$ and from the line wings that $m=0$. Both are consistent with Kallinger et al. (2008) but only the latter is consistent with De Ridder et al. (2006) and Barban et al. (2007).

The observed amplitude distribution of the 2nd frequency shows a clear dip in the centre, which is matched very closely by the $m=0$ fit, while the $m=-1$ fit lies just within the error in this region (centre top panel of Fig. 7). The $m=0$ fit also matches the phase distribution best at both the centre and in the wings, which implies that this is the most likely mode identification.

The asymmetry in the observed amplitude distribution of the 3rd frequency hampers a good fit, but in the line centre both $m=0$ and $m=-1$ fall well within the error bars (right top panel of Fig. 7). The centre part of the phase distribution is most closely fitted with the $m=-1$ fit, while the line wings are again best fitted with an $m=0$ fit. Thus here, the centre of the line favours a $m=-1$ mode identification, but $m=0$ is also possible.

For this star, the preferred inclination angles, computed from the weighted mean and standard deviation, all lie in the range $63 \pm 18^{\circ}$, which, together with the projected rotational velocity of $5.7 \mathrm{~km} \mathrm{~s}^{-1}$, results in an surface rotational frequency ranging between 0.8 and $1.1 \mu \mathrm{Hz}$.

\section{4. $\eta$ Serpentis}

After confirming that we can compare the line-profile variations from observations and synthetic spectra quantitatively, we analysed the CCFs of $\eta$ Serpentis. The results are listed in Table 2 and shown in Fig. 9. For these distributions, we see again that $m=-2$ and $m=2$ are the least favoured mode identification for all frequencies.

For $\eta$ Serpentis, all amplitude distributions are asymmetric and therefore none of the fits are consistent in the line wings, while in the centre both $m=0$ and $m=-1$ or $m=1$ are consistent with the observed distribution. In the centre of the phase distribution, the $m=1$ (1st and 2nd frequency) and $m=-1$ (3rd frequency) fits match the observations closely, while the $m=0$ fits are just within the errors (1st and 2nd frequency) or are even outside the error bars (3rd frequency). The phase distributions in the CCF wings are best matched by the $m=0$ fits. For this star, the line centres favour an $m=1$ (1st and 2nd frequency) or $m=-1$ (3rd frequency) mode identification, while $m=0$ cannot be ruled out because of the better fits in the CCF wings.

The inclination angles of the synthetic line profiles fitted to the data have a weighted mean value of $57^{\circ}$ with a standard deviation of $16^{\circ}$ (see Fig. 10 for the histogram). This range of inclination angles together with the projected rotational velocity determined with FAMIAS (Table 2) results in a surface rotational frequency between 1.5 and $2.1 \mu \mathrm{Hz}$.

\section{Discussion and conclusions}

To take the analysis of line-profile variations performed by Hekker et al. (2006) one step further, i.e., from a qualitative analysis to a quantitative analysis, it was necessary to apply a number of specific corrections to the cross-correlation functions. With these corrections we removed profiles with outliers at any velocity across the line profile. These outliers seem to have caused the large, single peaked amplitude profiles and low phase changes of the line-profile variations, most clearly seen in the dominant frequencies of $\epsilon$ Ophiuchi and $\eta$ Serpentis (see Figs. 6 and 7 of Hekker et al. 2006). Hekker et al. (2006) could match these amplitude profiles qualitatively with $m=2$ modes, but the low phase change over the profile could not be reproduced by synthetic line profiles, which hampered a definite mode identification. The corrections applied here altered the amplitude and phase distributions, and to these corrected distributions we could perform a quantitative analysis. 

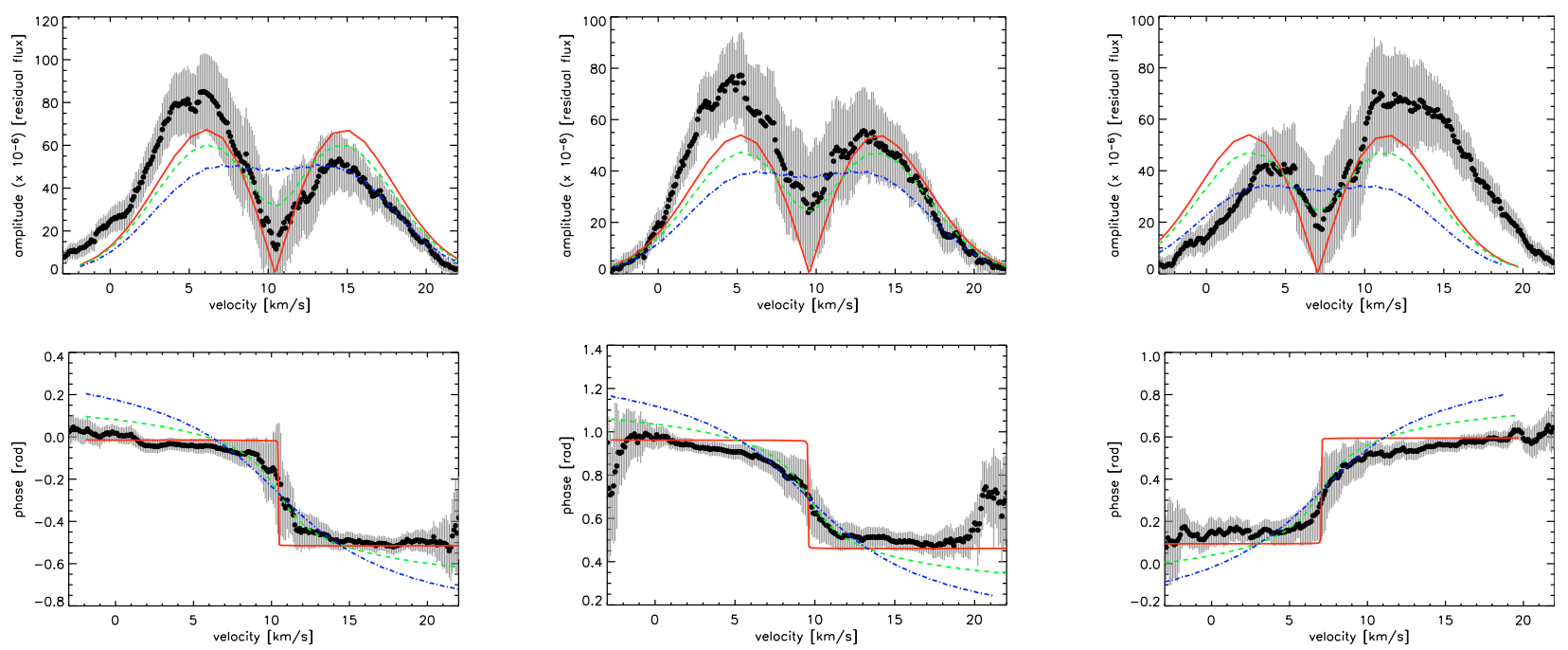

Fig. 9. Same as Fig. 5 for the significant frequencies of $\eta$ Serpentis obtained from $\langle\mathrm{v}\rangle, v_{\langle\mathrm{v}\rangle}=11.74 \mathrm{~cd}^{-1}(135.9 \mu \mathrm{Hz})(l e f t), v_{\langle\mathrm{v}\rangle}=9.48 \mathrm{~cd} \mathrm{~d}^{-1}$ $(109.7 \mu \mathrm{Hz})($ centre $)$, and $\nu_{\langle\mathrm{v}\rangle}=10.38 \mathrm{c} \mathrm{d}^{-1}(120.1 \mu \mathrm{Hz})($ right $)$. The mean radial velocity of the star is approximately $9.4 \mathrm{~km} \mathrm{~s}^{-1}$.

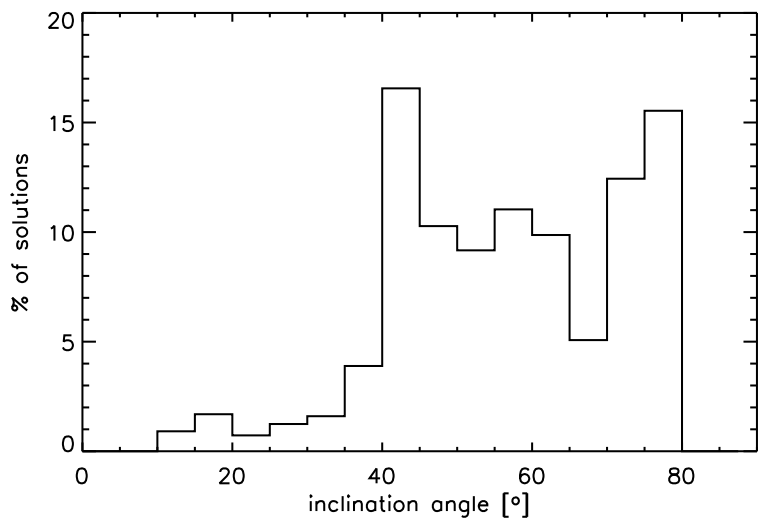

Fig. 10. Same as Fig. 4 for $\eta$ Serpentis.

Table 3. Intervals for the surface rotation frequencies $(\Omega)$ for the four evolved stars discussed here, together with the inclination angle $(i)$ and projected rotational velocity $(v \sin i$ ), we used to compute $\Omega$.

\begin{tabular}{lccc}
\hline \hline star & $\begin{array}{c}\Omega \\
\mu \mathrm{Hz}\end{array}$ & $\begin{array}{c}v \sin i \\
\text { degree }\end{array}$ & $\mathrm{km} \mathrm{s}^{-1}$ \\
\hline$\beta$ Hydri & $3.6-5.5$ & $38-72$ & 4.3 \\
$\epsilon$ Ophiuchi & $0.8-1.1$ & $45-81$ & 5.7 \\
$\eta$ Serpentis & $1.5-2.1$ & $41-73$ & 5.6 \\
$\delta$ Eridani & $3.1-4.9$ & $38-76$ & 4.9 \\
\hline
\end{tabular}

We have been able to analyse line-profile variations in the corrected CCFs of four evolved stars and identify the azimuthal order for the frequencies detected in the first moments with an uncertainty of \pm 1 as is usually the case in spectroscopic mode identification with the adapted method. From the synthetic line profile fitting of non-radial modes performed with FAMIAS, we could also determine a range of inclination angles for the stars. These together with the projected rotational velocity provided us with the surface rotational frequencies, which are all listed in Table 3.

The uncertainty in the mode identification is mostly due to different favourable identifications in the line wings and the line centres. Data with higher signal-to-noise ratio, i.e., with lower noise levels, than we have at hand could improve the mode identification significantly. Firstly, this higher signal-to-noise ratio would decrease the asymmetry in the observed amplitude distributions for modes with $m=0$, as seen from simulations. Secondly, lower noise values could reduce the errors on the amplitude and phase distributions, which will allow us to better distinguish between fits with different $m$ values. Nevertheless, it is possible that higher signal-to-noise ratio of the data will not improve the fits to the phase distribution. It is clear from the lower panels of Figs. 5, 7, and 9 that for $m \neq 0$ the phase difference in the wings changes gradually for the synthetic profiles, while this is less so for the observed profiles. We tested whether this discrepancy was due to the fact that we neglect temperature effects on the equivalent width, but taking these into account did not improve the fitting considerably.

For $\beta$ Hydri, the azimuthal orders obtained from the lineprofile analysis are compatible with the harmonic degree of the modes determined previously from radial velocity measurements and asymptotic frequency relations by Bedding et al. (2007). Three modes with $\ell=0$ and one mode with $\ell=1$ are compatible with $m=0$, while for the fifth frequency we know $\ell=1$ and $m=-1$ is clearly favourable in this case.

The mode identifications for $\delta$ Eridani are also consistent with the results from Carrier (2002), Carrier et al. (2003), although the present method infers that the dominant frequency is a non-radial mode instead of a radial one, as claimed by Carrier (2002), Carrier et al. (2003). Our identification would change the degrees assigned to the different ridges in the échelle diagram, but we do not have enough frequencies for which we could apply the current method to make any firm statements.

For $\epsilon$ Ophiuchi we have found only one mode in common with the frequency model fitting performed by Kallinger et al. (2008). The identifications for this non-radial oscillation mode are also in agreement. As we find two possible values of the azimuthal order for all frequencies, our analysis could also be in agreement with the radial mode interpretation favoured by De Ridder et al. (2006) and Barban et al. (2007).

The confirmation that we can compare line-profile variations of observations and synthetic profiles quantitatively led us to also analyse $\eta$ Serpentis. Also for this star, we were able to obtain the azimuthal orders for three modes with an uncertainty of \pm 1 and an indication of the surface rotational frequency. These identifications and the surface rotational frequency are less accurate 
due to the lower $\mathrm{S} / \mathrm{N}$ of the amplitude and phase diagrams, which are therefore more asymmetric than for the other stars.

With FAMIAS, we fitted $v \sin i$ independent of known literature values. For all four stars, we found higher values than current literature values (see Tables 1 and 2). The difference in the quoted values might be related to differences in equivalent width and $\xi_{\text {macro }}$.

As discussed here, improvements in the spectral line profile analysis are still needed, both in terms of higher signal-to-noise ratio observations and in terms of the generated synthetic profiles we compare the data with. In the future we hope to expand on an analysis of simulated data, as already started by Hekker et al. (2006). We also want to apply the method to evolved stars with solar-like oscillations observed using data of high signalto-noise ratio, which we expect to become available from for instance SONG (Stellar Observations Network Group). We anticipate that this will improve our understanding and increase the value of this method for mode identification in solar-like oscillators.

Acknowledgements. S.H. wants to thank Maarten Mooij for useful discussions, Wolfgang Zima for his help with FAMIAS and Anne-Marie Broomhall for her help with language editing. S.H. acknowledges financial support from the Belgian Federal Science Policy (ref: MO/33/018) and the UK Science and Technology Facilities Council. The research leading to these results has received funding from the European Research Council under the European Community's Seventh Framework Programme (FP7/2007-2013)/ERC grant agreement $n^{\circ} 227224$ (PROSPERITY), as well as from the Research Council of K. U. Leuven grant agreement GOA/2008/04. We would like to thank our referee for valuable comments, which helped to improved the manuscript considerably.

\section{References}

Aerts, C., Christensen-Dalsgaard, J., \& Kurtz, D. W. 2009, Asteroseismology (Springer-Verlag), in press

Aerts, C., De Pauw, M., \& Waelkens, C. 1992, A\&A, 266, 294

Aerts, C., \& Eyer, L. 2000, in Delta Scuti and Related Stars, ed. M. Breger, \& M. Montgomery, ASP Conf. Ser., 210, 113
Baranne, A., Queloz, D., Mayor, M., et al. 1996, A\&AS, 119, 373

Barban, C., De Ridder, J., Mazumdar, A., et al. 2004, in SOHO 14 Helio- and Asteroseismology: Towards a Golden Future, ESA SP-559, 113

Barban, C., Matthews, J. M., de Ridder, J., et al. 2007, A\&A, 468, 1033

Bedding, T. R., Huber, D., Stello, D., et al. 2010, ApJ, 713, L176

Bedding, T. R., Kjeldsen, H., Arentoft, T., et al. 2007, ApJ, 663, 1315

Briquet, M., Lefever, K., Uytterhoeven, K., \& Aerts, C. 2005, MNRAS, 362, 619

Briquet, M., Uytterhoeven, K., Morel, T., et al. 2009, A\&A, 506, 269

Carrier, F. 2002, Ph.D. Thesis, Observatoire de Genève Maullettes, 1290 Sauverny, Switzerland

Carrier, F., Bouchy, F., \& Eggenberger, P. 2003, in Asteroseismology Across the HR Diagram, ed. M. J. Thompson, M. S. Cunha, \& M. J. P. F. G. Monteiro, 311

De Ridder, J., Barban, C., Carrier, F., et al. 2006, A\&A, 448, 689

De Ridder, J., Barban, C., Baudin, F., et al. 2009, Nature, 459, 398

Desmet, M., Briquet, M., Thoul, A., et al. 2009, MNRAS, 396, 1460

Dravins, D., Lindegren, L., \& Vandenberg, D. A. 1998, A\&A, 330, 1077

Dupret, M., Belkacem, K., Samadi, R., et al. 2009, A\&A, 506, 57

Dziembowski, W. A., Gough, D. O., Houdek, G., \& Sienkiewicz, R. 2001, MNRAS, 328, 601

Frandsen, S., Carrier, F., Aerts, C., et al. 2002, A\&A, 394, L5

Gizon, L., \& Solanki, S. K. 2003, ApJ, 589, 1009

Hekker, S., \& Meléndez, J. 2007, A\&A, 475, 1003

Hekker, S., Aerts, C., de Ridder, J., \& Carrier, F. 2006, A\&A, 458, 931

Houdek, G., \& Gough, D. O. 2002, MNRAS, 336, L65

Kallinger, T., Guenther, D. B., Matthews, J. M., et al. 2008, A\&A, 478, 497

North, J. R., Davis, J., Bedding, T. R., et al. 2007, MNRAS, 380, L80

Perryman, M. A. C., \& ESA 1997, The HIPPARCOS and TYCHO catalogues. Astrometric and photometric star catalogues derived from the ESA HIPPARCOS Space Astrometry Mission, ESA SP, 1200

Reiners, A., \& Schmitt, J. H. M. M. 2003, A\&A, 398, 647

Richichi, A., Percheron, I., \& Khristoforova, M. 2005, A\&A, 431, 773

Rupprecht, G., Pepe, F., Mayor, M., et al. 2004, in SPIE Conf. Ser. 5492, ed. A. F. M. Moorwood, \& M. Iye, 148

Santos, N. C., Israelian, G., \& Mayor, M. 2004, A\&A, 415, 1153

Tassoul, M. 1980, ApJS, 43, 469

Telting, J. H., Aerts, C., \& Mathias, P. 1997, A\&A, 322, 493

Thévenin, F., Kervella, P., Pichon, B., et al. 2005, A\&A, 436, 253

Valenti, J. A., \& Fischer, D. A. 2005, ApJS, 159, 141

Zima, W. 2006, A\&A, 455, 227

Zima, W. 2008, Commun. Asteroseismol., 155, 17 